\title{
Trend Analysis of Head and Neck Neoplasms between 2012-2018 in Patients Residing in Al-Madinah, Saudi Arabia: A Retrospective Study
}

\author{
Maha T. Alsharif ${ }^{1} \quad$ Alla T. Alsharif ${ }^{2}$ Majid A. Krsoum ${ }^{3,4}$ Mazen A. Aljohani ${ }^{3}$ Osama M. Qadiri3,4 \\ Abdulkarim A. Alharbi ${ }^{3,5}$ Sadeq A. Al-Maweri, ${ }^{6,7}$ Saman Warnakulasuriya ${ }^{8,9}$ Saba Kassim²
}

10ral Diagnostic Sciences Department, Faculty of Dentistry, King Abdulaziz University, Jeddah, Saudi Arabia

2 Preventive Dental Sciences, Taibah University Dental College and Hospital, Al-Madinah Al-Munawwarah, Saudi Arabia

${ }^{3}$ Taibah University Dental College and Hospital, Al-Madinah

Al-Munawwarah, Saudi Arabia

4Prince Mohammed bin Abdulaziz Hospital, Ministry of National Guard - Health Affairs, Al-Madinah Al-Munawwarah, Saudi Arabia

${ }^{5}$ Ministry of Health, Salah Al-Din Primary Health Center, Ha'il,

Saudi Arabia

${ }^{6}$ Department of Oral Medicine and Diagnostic Sciences, AlFarabi Colleges of Dentistry and Nursing, Riyadh, Saudi Arabia

\begin{abstract}
Address for correspondence Saba Kassim, PhD, Preventive Dental Sciences, Taibah University Dental College and Hospital, Al-Madinah Al-Munawwarah, 42353 Saudi Arabia (e-mail: saba262003@gmail.com).
${ }^{7}$ Department of Oral Medicine, Oral Pathology and Oral Radiology Faculty of Dentistry, Sana'a University, Sana'a, Yemen
8 Faculty of Dentistry, Oral \& Craniofacial Sciences, King's College London, London, United Kingdom
${ }^{9}$ World Health Organization Collaborating Centre for Oral Cancer, London, United Kingdom

\begin{abstract}
Keywords

- head and neck neoplasms

- trend analysis

- diagnostic delays

- histopathology

- Saudi Arabia

Objectives This study sought to present a view of head and neck neoplasms (HNN) prevalence with specific focus on sociodemographic determinants in Al-Madinah Province, Saudi Arabia.

Materials and Methods This was a hospital-based retrospective study based on retrieval of histopathological data for a period of 6 years between 2012 and 2018. Data was collected from the archives of the Oral and Maxillofacial Pathology Laboratory at King Fahad Hospital (the only referral center for biopsy services) in Al-Madinah City.

Statistical Analysis An independent $t$-test and/or nonparametric (Mann-Whitney $U$ test, chi-squared test) tests were used to determine the differences between groups. Statistical significance was set at the $p$-value $<0.05$.

Results Out of 96 patients, a total of 58 patients had valid biopsy data with preoperative diagnosis. Over three quarters of the cases $(n=44)$ were benign with only $24 \%$ were malignant. Males were more likely to be diagnosed with a benign tumor than females ( 54.5 vs. $45.6 \%$, respectively), and malignancy was also more common in males ( 64.3 vs. 24.1). A significant difference was found in relation to mean age of older patients who were more likely to be diagnosed with malignant tumors $(p=0.001)$.

Conclusions The findings suggest that most of biopsied HNN cases are benign neoplasms. Age is a significant risk factor for head and neck malignancy in this region. Delays in diagnosis of HNN need to be explored.
\end{abstract}

published online

March 10, 2021
DOI https://doi.org/

$10.1055 / \mathrm{s}-0040-1722090$ ISSN 1305-7456. (c) 2021. European Journal of Dentistry.

This is an open access article published by Thieme under the terms of the Creative Commons Attribution License, permitting unrestricted use, distribution, and reproduction so long as the original work is properly cited. (https://creativecommons. org/licenses/by/4.0/)

Thieme Medical and Scientific Publishers Pvt. Ltd., A-12, 2nd Floor,

Sector 2, Noida-201301 UP, India 


\section{Introduction}

Head and neck neoplasms (HNN) include a heterogeneous group of tumors, both benign and malignant predominantly affecting the upper aerodigestive track. ${ }^{1}$ In the United States, head and neck cancer accounts for $3 \%$ of all cancers. ${ }^{2}$ Based on the data collated and compiled at the International Agency for Research on Cancer, anatomical sites including the lip, tongue, and mouth with 355,000 newly diagnosed cases per year could be considered to carry a high proportion of head and neck cancers. ${ }^{3}$ Among the head and neck cancers (HNC), the epidemiology of oral cancer has almost exclusively been studied in Saudi population where risk factors vary widely in different regions. ${ }^{4-6}$ However, very little is known about the magnitude of the risk factors, morbidity and mortality of HNN (benign and malignant) in Saudi Arabia. ${ }^{6}$ In the context of different geographic locations, knowledge about the HNN distribution and its variability within certain community profile (i.e., gender and age) is an essential prerequisite. The current literature elucidates noticeable regional differences in patterns of incidence of HNN in Saudi Arabia. ${ }^{7-10}$ This geographic variation could be attributed to cultural differences and associated risk factors. ${ }^{11}$ Availability of such data can help in prioritization and provision of population-specific healthcare services, implementation of preventive strategies, and distribution of resources according to specific population needs. ${ }^{12}$

Saudi Arabia is one of the largest Arab countries of the Arabian Peninsula that is divided into 6 regions and 13 provinces. $^{13}$ The highest incidence rate of HNC (oral cancer) was reported in the southern province of Jazan, Saudi Arabia. In Jazan, oral squamous cell carcinoma is currently the most common malignancy diagnosed in females and second-most common in males. ${ }^{14}$ Use of smokeless tobacco (shamma) has been a recognized risk factor for the high incidence of oral cancer in women in this region. ${ }^{14}$ In another study, by Al-Zahrani et al, investigating outpatient palliative care at a major tertiary hospital in Saudi Arabia, HNC was the second most common cancer among patients (15.3\%). ${ }^{5}$ However, in the northern region, benign conditions were diagnosed more frequently than malignancies. ${ }^{9}$ Al-Madinah Province is located in the western region of Saudi Arabia, with a population of 2,132,679. ${ }^{13}$ Since 2009, King Fahad Hospital in Al-Madinah City is the only specialized referral center that offers free oral and maxillofacial consultative and diagnostic services throughout Al-Madinah Province and other peripheral hospitals in the region. The hospital offers aforementioned healthcare services to all patients that minimizes access bias contributing to greater accuracy on the incidences of neoplasms in this region. Due to the lack of information regarding the prevalence of HNN in Al-Madinah Province, the present study aimed to present data on HNN prevalence with specific focus on sociodemographic determinant in Al-Madinah Province, Saudi Arabia.

\section{Materials and Methods}

\section{Study Design and Subjects}

This was a retrospective study based on the primary data retrieved from the archives of the Oral and Maxillofacial Pathology Laboratory at King Fahad Hospital in Al-Madinah City.

All cases with confirmed histopathology analysis of a HNN (ICD-10: C00-C44, D00-D23) over a period of 6 years (20122018) were included in the study. Patients who were diagnosed with jaw cysts, inflammatory lesions, reactive lesions, or those with no histopathological diagnosis were excluded.

\section{Data Collection}

The data collection sheet was designed by Microsoft Office Excel 2016 to enter information from King Fahad Hospital's archives. All data were collected and checked by two investigators. Electronic search was generated first, then manual extraction of the data was done to make sure no data were missed. A temporary user platform was created by the hospital's IT department with restricted access to identifiable health information for protection of patients' privacy. Other information included the following: demographic data (sex, age and nationality), date of admission to the hospital with length of stay, preoperative diagnosis, histological diagnosis, and biopsy site. Data related to presence of multiple lesions of a patient was recorded based on the history of recurrent lesions or two or more lesions on different sites of the same patient. All of the biopsies were performed by oral and maxillofacial surgeons and submitted for histopathological examination by certified general pathologists at King Fahad Hospital.

Data collection sheet involved two parts: the first part was a collection of demographic data and preoperative diagnoses, including all patients with lesions, tumors, neoplasms, cancer, and some metastasized conditions. The second part was a collection of final histopathological diagnoses according to the biopsies undertaken. Both incisional and excisional biopsies were included, and if the same patient had an incisional and excisional biopsy, the excisional biopsy was counted. Sociodemographic differences between malignant (C00-C44) and benign (D00-D23) neoplasms were assessed. The anatomic sites were further categorized into the four most common locations; the other locations were grouped under "others."

\section{Statistical Analysis}

The data were imported from excel sheet into the Statistical Package for Social Sciences (SPSS Inc.; Chicago, Illinois, United States) version 20 for analysis. Descriptive analysis was performed to report the demographic variables of the patients. Means and standard deviations were reported for the continuous variables with normal distribution, and as median and Interquartile when no adherence to normality was observed (Kolmogorov-Smirnov, $p<0.05$ ). Frequencies and percentages $[F(\%)]$ were used for categorical variables. An independent $t$-test and/or nonparametric (Mann-Whitney $\mathrm{U}$ test, chi-squared test) tests were used to determine the 
differences between groups. Statistical significance was set at the $p$-value $<0.05$.

\section{Ethical Consideration and Confidentiality}

The protocol for the study was approved by Taibah University's Dental College Ethical Research Committee (IRB approval no. TUCDREC/20170920/Alsharif) and the Ministry of Health (approval no. IRB-116), Al-Madinah, Saudi Arabia. Due to the retrospective nature of the study, patients' informed consent was not required. However, confidentiality of the information was assured, that is, every patient was assigned a code.

\section{Results}

\section{Patients' Characteristics}

Out of 96 patients, a total of 58 patients had valid biopsy data with preoperative diagnosis and final histopathology diagnosis of a HNN. A total of $58 \mathrm{HNN}$ cases met the inclusion criteria. - Table 1 shows the characteristics of the subjects: the majority (70\%) were of Saudi nationality with a slight male predominance (56.9\%). The mean age of the patients was $\sim 39$ \pm 19 years old. Multiple head and neck lesions were found in four $(6.9 \%)$ of the subjects. On average, the patients remained in the hospital for 3 days after undergoing a biopsy. - Table 1 also shows that 9 patients (15.5\%) were readmitted to King Fahad hospital after being discharged.

- Table 2 also illustrates that males were more likely to be diagnosed with a benign tumor than females ( 54.5 vs. $45.6 \%$, respectively), and malignancy was also more common in males ( 64.3 vs. $24.1 \%$ ). The mean time between the first examination and final diagnosis for all lesions is found in - Table 2 . The average time frame of "door to diagnosis" for patients diagnosed with benign lesions was $\sim 42.3 \pm 67.7$ days and for malignant $29.9 \pm 30.8$ days.

\section{Tumors' Sites and Trend Over Time}

As shown in - Fig. 1, the most frequently affected anatomical site was the mandible ( $n=19,32.8 \%$ ), with $94.7 \%$ of benign lesions followed by the parotid gland ( $n=9,15.5 \%)$, palate and facial skin $(n=5,8.6 \%)$.

Table 1 Characteristics of patients with head and neck neoplastic lesions $(n=58)$

\begin{tabular}{|l|l|l|}
\hline \multicolumn{2}{|l|}{ Characteristics } & Frequency (\%) \\
\hline \multirow{2}{*}{ Gender } & Males & $33(56.9)$ \\
\cline { 2 - 3 } & Females & $25(43.1)$ \\
\hline \multirow{2}{*}{ Nationality } & Saudi & $40(69)$ \\
\cline { 2 - 3 } & $\begin{array}{l}\text { Non- } \\
\text { Saudi }\end{array}$ & $18(31)$ \\
\hline Multiple sites & Yes & $4(6.9)$ \\
\cline { 2 - 3 } & No & $54(93.1)$ \\
\hline Readmission & Yes & $9(15.5)$ \\
\cline { 2 - 3 } & No & $49(84.5)$ \\
\hline $\begin{array}{l}\text { Length of stay (d) } \\
\text { Median (IQR) }\end{array}$ & $3(4.3)$ \\
\hline
\end{tabular}

Abbreviation: IQR, interquartile range.
Trend analysis of the data commencing in 2012 showed an increased incidence of head and neck neoplastic lesions in 2013/14 (-Fig. 2), with subsequent gradual decline in prevalence. A sharp increase in the number of new cases was observed from 7 cases to 15 cases per year from 2016 to 2018 but regarded statistically nonsignificant $(p>0.05)$.

\section{Bivariate Analyses of Factors in Relationship to Malignant and Benign Lesions}

In bivariate analyses, some of the variables revealed statistically significant differences $(p<0.05)$ in relation to lesion diagnosis (-Table 3 ). A significant difference was found in relation to mean age of older patients who were more likely to be diagnosed with malignant tumors $(p=$ 0.001 ). The hospital stay of patients with malignant lesions was extended beyond that of those with benign lesions $(p=0.016)$.

\section{Discussion}

This study examined the trends in the prevalence of HNN using the data from a tertiary care facility for the period 2012 to 2018. Besides, a predictive analysis was conducted on this dataset to determine the impact of sociodemographic factors and under reporting of HNN. To the best of our knowledge, this was the first study that assessed the trend of HNN in Al-Madinah Province and prevalence of HNN has not previously been reported. Most of the prevalence studies were mainly conducted in the Southern or Eastern provinces and were either limited to oral cancer ${ }^{6}$ or to the study of clinical cases. ${ }^{15}$ There is also paucity of data that describe both benign and malignant neoplasms of the head and neck in descriptive studies in the world literature. ${ }^{16}$

Overall, the results of the present study showed that the majority of diagnosed HNN cases were benign neoplasms (particularly, pleomorphic adenoma and ossifying fibroma), while only 14 cases were malignant (mainly, squamous cell carcinoma). These findings are in line with previous studies conducted in other regions of Saudi Arabia. ${ }^{8,9,17}$ However, this finding is different from that reported in Jazan, Saudi Arabia, where 38\% of cases were malignant. ${ }^{7}$ The high incidence of malignant cancers in the latter study can be attributed to the high prevalence of smokeless tobacco use (a wellknown risk factor for oral cancer) in South-western part of Saudi Arabia. ${ }^{18,19}$ However, the prevalence of cigarette smoking in Al-Madinah was $21.3 \%$ among adolescent males ${ }^{20}$ and $9.8 \%$ among college-aged females. ${ }^{21}$ In Saudi, there has been rapid growth in current electronic cigarette use over the past few years, particularly among young adults..$^{22}$ This rapid increase in tobacco use would have a significant impact on the future burden of disease in this region. E-cigarettes pose serious health hazards, including increased risk for heart and respiratory disease but its causal effects on human cancer would not 
Table 2 Distribution of head and neck neoplasm based on lesions ( $n=58)$

\begin{tabular}{|c|c|c|c|c|}
\hline \multirow[t]{2}{*}{ Head and neck lesions } & \multirow[t]{2}{*}{$n(\%)$} & \multicolumn{2}{|c|}{ Gender, $n(\%)$} & \multirow[t]{2}{*}{ Age range/years } \\
\hline & & Male & Female & \\
\hline \multicolumn{5}{|l|}{ Benign neoplasms } \\
\hline Pleomorphic adenoma & $7(12.1)$ & $3(42.9)$ & $4(57.1)$ & $24-59$ \\
\hline Ossifying fibroma & $7(12.1)$ & $4(57.1)$ & $3(42.9)$ & $6-36$ \\
\hline Ameloblastoma & $5(8.6)$ & $4(80)$ & $1(20)$ & $28-60$ \\
\hline Warthin tumor & $4(6.9)$ & $2(50)$ & $2(50)$ & $26-81$ \\
\hline Odontogenic myxoma & $4(6.9)$ & $3(75)$ & $1(25)$ & $14-26$ \\
\hline Acquired melanotic nevus & $2(3.4)$ & $2(100)$ & $0(0)$ & $35-63$ \\
\hline Central giant cell lesion & $2(3.4)$ & $1(50)$ & $1(50)$ & $17-35$ \\
\hline Squamous papilloma & $2(3.4)$ & $2(100)$ & $0(0)$ & 27 \\
\hline Lipoma & $2(3.4)$ & $1(50)$ & $1(50)$ & $41-53$ \\
\hline Cementoma & $1(1.7)$ & $0(0)$ & $1(100)$ & 57 \\
\hline Solitary fibrous tumor & $1(1.7)$ & $0(0)$ & $1(100)$ & 64 \\
\hline Pilomatricoma & $1(1.7)$ & $0(0)$ & $1(100)$ & 5 \\
\hline Lobular capillary hemangioma & $1(1.7)$ & $0(0)$ & $1(100)$ & 40 \\
\hline Cavernous hemangioma & $1(1.7)$ & $0(0)$ & $1(100)$ & 10 \\
\hline Unspecified benign neoplasm & $4(6.9)$ & $2(50)$ & $2(50)$ & $25-54$ \\
\hline Total $(\%) /$ mean \pm SD & $44(75.9)$ & $24(54.5)$ & $20(45.6)$ & $5-81$ \\
\hline \multicolumn{5}{|l|}{ Malignant neoplasms } \\
\hline Squamous cell carcinoma & $5(8.5)$ & $2(40)$ & $3(60)$ & $36-66$ \\
\hline Mucoepidermoid carcinoma & $3(5.2)$ & $3(100)$ & $0(0)$ & $17-62$ \\
\hline Metastatic carcinoma & $2(3.4)$ & $1(50)$ & $1(50)$ & $79-81$ \\
\hline Osteosarcoma & $1(1.7)$ & $0(0)$ & $1(100)$ & 28 \\
\hline $\begin{array}{l}\text { Malignant neoplasm of maxillary } \\
\text { sinus }\end{array}$ & $1(1.7)$ & $1(100)$ & $0(0)$ & 61 \\
\hline Non-Hodgkin lymphoma & $1(1.7)$ & $1(100)$ & $0(0)$ & 69 \\
\hline Verrucous carcinoma & $1(1.7)$ & $1(100)$ & $0(0)$ & 57 \\
\hline Total $(\%) /$ mean \pm SD & $14(24.1)$ & $9(64.3)$ & $5(35.7)$ & $17-81$ \\
\hline
\end{tabular}

Abbreviation: SD, standard deviation.

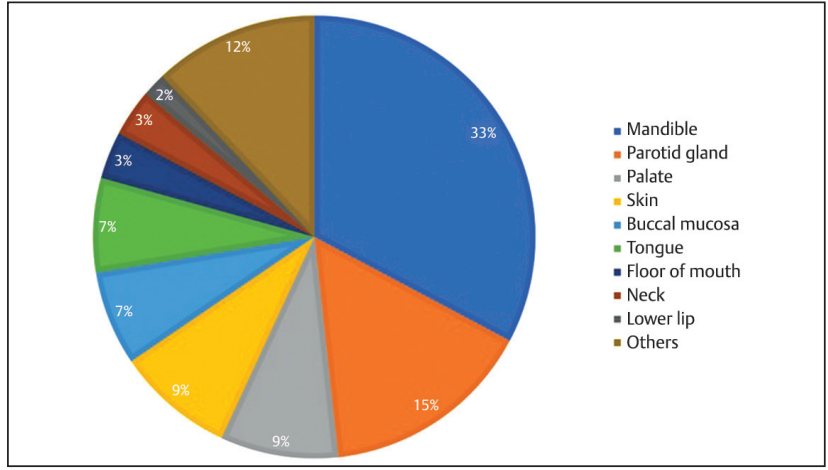

Fig. 1 Distribution of head and neck tumors based on anatomic location.

be known for a few decades. Themes commonly used in antismoking messages may be effective in educating the public about the potential harm of e-cigarettes. ${ }^{23}$

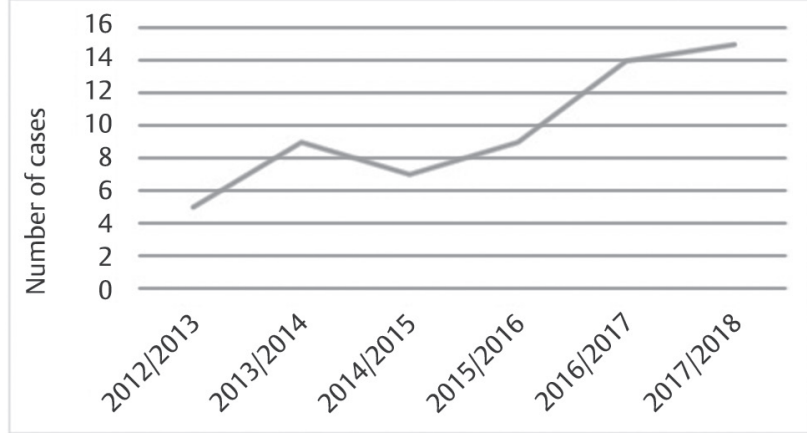

Fig. 2 Incidence for head and neck neoplasms over the period 2012 to 2018 .

Additionally, the mandible was the most frequently affected site for benign lesions, followed by the parotid gland. Intercountry comparisons revealed contradicting results citing lip and buccal mucosa as common sites for benign lesions. ${ }^{4}$ 
Table 3 Characteristics and bivariate analysis results of comparisons of patients with benign $(n=44)$ and malignant lesions $(n=14)$

\begin{tabular}{|c|c|c|c|c|}
\hline \multicolumn{2}{|c|}{ Patients' characteristics } & \multicolumn{2}{|c|}{ Diagnosis: $n$ (\%) } & \multirow[t]{2}{*}{$p$-Value } \\
\hline & & Benign lesion & Malignant lesion & \\
\hline \multirow[t]{2}{*}{ Gender } & Males & $24(72.7)$ & $9(27.3)$ & \multirow[t]{2}{*}{0.52} \\
\hline & Females & $20(80)$ & $5(20)$ & \\
\hline \multirow[t]{2}{*}{ Nationality } & Saudi & $32(80)$ & $8(20)$ & \multirow[t]{2}{*}{0.27} \\
\hline & Non-Saudi & $12(66.7)$ & $6(33.3)$ & \\
\hline \multirow[t]{2}{*}{ Multiple sites } & Yes & $9(100)$ & $0(0)$ & \multirow[t]{2}{*}{0.06} \\
\hline & No & $35(71.4)$ & $14(28.6)$ & \\
\hline \multirow[t]{2}{*}{ Readmission } & Yes & $7(77.8)$ & $2(22.2)$ & \multirow[t]{2}{*}{0.88} \\
\hline & No & $37(75.9)$ & $12(24.1)$ & \\
\hline \multirow[t]{5}{*}{ Site } & Mandible & $18(94.7)$ & $1(5.3)$ & \multirow[t]{5}{*}{0.058} \\
\hline & Parotid gland & $6(66.7)$ & $3(33.3)$ & \\
\hline & Palate & $3(60)$ & $2(40)$ & \\
\hline & Skin & $5(100)$ & $0(0)$ & \\
\hline & Others & $12(60)$ & $8(40)$ & \\
\hline & & \multicolumn{2}{|c|}{ Diagnosis: mean $\pm S D$} & \multirow[t]{2}{*}{$p$-Value } \\
\hline & & Benign lesion & Malignant lesion & \\
\hline \multicolumn{2}{|c|}{ Age (y) } & $33.9 \pm 17.4$ & $55.3 \pm 18.5$ & 0.001 \\
\hline \multicolumn{2}{|c|}{ Length of stay $(\mathrm{d})$} & $4.4 \pm 5.9$ & $5.4 \pm 2.9$ & 0.016 \\
\hline \multicolumn{2}{|c|}{ Time from door-to-diagnosis (d) } & $42.3 \pm 67.7$ & $29.9 \pm 30.8$ & 0.610 \\
\hline
\end{tabular}

Abbreviation: SD, standard deviation.

Pleomorphic adenoma was one the most common tumors found among benign neoplasms. ${ }^{24}$ Among salivary gland neoplasms similar findings were reported by Tian et al..$^{25}$ The etiology of salivary gland tumors is unknown and they comprise $3 \%$ of head and neck tumors in the United States. ${ }^{26}$

A significant difference was found in relation to mean age of patients with the older people who were more likely to be diagnosed with malignant tumors. This finding consolidates the findings of previous studies. ${ }^{18,27}$ Age is a known risk factor for head and neck malignancies. ${ }^{28}$ Like many developing nations, Al-Madinah population age-structure is most of younger cohorts. ${ }^{10}$ This composition has a significant impact on the present pattern/type of HNN and possible future trends. With modernization, this demographic transition is certainly underway, and the total number of people will increase as will the proportion of older people. Subsequently, the cancer burden that mainly affect the elderly will also grow.

The mean interval from door-to-diagnosis for malignant lesions was estimated close to 30 days. This delay is considered unacceptable and efforts should be taken to explore the reasons for diagnostic delay and to bridge the gaps in getting the diagnostic service to report any malignancy within 2 weeks. There is a need for primary care studies on oral cancer diagnosis. The paucity of primary care research in this area in the global literature was recently highlighted by Grafton-Clarke. ${ }^{29}$

As stated above, this is one of the few studies that documented HNN neoplasms in Saudi Arabia. This study emphasizes the importance of oral health promotion and disease prevention programs that is specifically designed to improve oral health literacy and the quality of life of the population in this region. However, the study has some limitations worth highlighting. This was a retrospective, hospital-based study and some essential data were lacking (e.g., different modifiable and nonmodifiable risk factors that contribute to HNN such as socioeconomic status, tobacco, oral hygiene, nutritional factors, inherited syndromes, and immunological conditions)..$^{30,31}$ Importantly, the nationality of the patients would be underpinned with further investigation of ethnicity/origin as this might influence the HNN. This is based on that many Saudi nationals are of different ethnicity/origin backgrounds. Also, the number of the studied sample is considered relatively low as only clinically suspected tumors are referred for histopathological examination. Alternatively, limited awareness or late biopsy seeking behavior for HNN or the paucity of specialties in the required fields may be the reason for this low histopathologic reporting. Furthermore, referred patients may have sought care elsewhere as the maxillofacial pathology service is just recently established at King Fahad Hospital in Al-Madinah. In addition, the representativeness of the study findings to whole population of the Al-Madinah is questionable as lifestyle risk factors and treatment seeking behaviors may differ by the regions. Out of 96 patients, a total of 58 patients had valid biopsy data with preoperative diagnosis. Therefore, there is a need to biopsy all suspected neoplastic conditions, as recommended in other Saudi Arabian studies. ${ }^{7}$

\section{Conclusion}

When a primary care physician or a dentist is confronted with a head and neck tumor, the adage "common things happen 
commonly" should be applied. We report here the frequency of head and neck tumors found in a pathology service that would be useful for improving referral services in this region of Saudi Arabia. Diagnostic delays encountered demand strengthening of guidelines for referral of suspected cancers in the community.

\section{Conflict of Interest}

None declared.

\section{Acknowledgment}

We would like to thank the King Fahad Hospital in Al-Madinah for facilitating data collection.

\section{References}

1 Mehanna H, Paleri V, West CM, Nutting C. Head and neck cancer-Part 1: epidemiology, presentation, and prevention. BMJ 2010;341:c4684

2 Chow LQM. Head and neck cancer. N Engl J Med 2020; 382(1):60-72

3 Miranda-Filho A, Bray F. Global patterns and trends in cancers of the lip, tongue and mouth. Oral Oncol 2020;102:10455110. Doi:1016/j.oraloncology.2019.104551

4 Alhazzazi TY. Evaluation of head and neck cancer awareness and screening status in Jeddah, Saudi Arabia. Asian Pac J Cancer Prev 2016;17(3):1135-1139

5 Al-Zahrani O, Eldali A, Al-Shahri MZ. Prevalence and severity of pain in cancer patients in an outpatient palliative care setting in Saudi Arabia. Qatar Med J 2014;2014(1):38-45

6 Alhazzazi TY, Alghamdi FT. Head and neck cancer in Saudi Arabia: a systematic review. Asian Pac J Cancer Prev 2016; 17(8):4043-4048

7 Saleh SM, Idris AM, Vani NV, et al. Retrospective analysis of biopsied oral and maxillofacial lesions in South-Western Saudi Arabia. Saudi Med J 2017;38(4):405-412

8 Alhindi NA, Sindi AM, Binmadi NO, Elias WY. A retrospective study of oral and maxillofacial pathology lesions diagnosed at the Faculty of Dentistry, King Abdulaziz University. Clin Cosmet Investig Dent 2019;11:45-52

9 Yousef Musarrah A, Mohammad Raji A, Khurram L, Nasser Attallah A, Badar Awadh A, Mohammed Ahmed A. A 5-years retrospective study oral pathological lesions in 425 Saudi patient. Pak Oral Dent J 2016;36(1):45

10 Al Yamani AO, Al Sebaei MO, Bassyoni LJ, Badghaish AJ, Shawly HH. Variation of pediatric and adolescents head and neck pathology in the city of Jeddah: a retrospective analysis over 10 years. Saudi Dent J 2011;23(4):197-200

11 Krishna Rao SV, Mejia G, Roberts-Thomson K, Logan R. Epidemiology of oral cancer in Asia in the past decade-an update (2000-2012). Asian Pac J Cancer Prev 2013;14(10):5567-5577

12 Gambhir RS, Veeresha KL, Sohi R, Kakkar H, Aggarwal A, Gupta D. The prevalence of oral mucosal lesions in the patients visiting a dental school in Northern India in relation to sex, site and distribution: a retrospective study. J Clin Exp Dent 2011;3(1):e10-e17. Doi:10.4317/jced.3.e10

13 General Authority of Statistics GAS. Population indicators data base 2020. Available at: http://87.101.205.104:6855/en/ DataAnalysis. Accessed November 24, 2020
14 Brown A, Ravichandran K, Warnakulasuriya S. The unequal burden related to the risk of oral cancer in the different regions of the Kingdom of Saudi Arabia. Community Dent Health 2006;23(2):101-106

15 Al-Mobeeriek A, AlDosari AM. Prevalence of oral lesions among Saudi dental patients. Ann Saudi Med 2009;29(5):365-368

16 Dilley DC, Siegel MA, Budnick S. Diagnosing and treating common oral pathologies. Pediatr Clin North Am 1991;38(5): 1227-1264

17 Ali M, Joseph B, Sundaram D. Prevalence of oral mucosal lesions in patients of the Kuwait University Dental Center. Saudi Dent J 2013;25(3):111-118

18 Quadri MFA, Alharbi F, Bajonaid AMS, Moafa IHY, Sharwani AA, Alamir AH. Oral squamous cell carcinoma and associated risk factors in Jazan, Saudi Arabia: a hospital based case control study. Asian Pac J Cancer Prev 2015;16(10):4335-4338

19 Al-Maweri SA, Al-Jamaei A, Saini R, Laronde DM, Sharhan A. White oral mucosal lesions among the Yemeni population and their relation to local oral habits. J Investig Clin Dent 2018;9(2):e12305

20 Al-Zalabani A, Kasim K. Prevalence and predictors of adolescents' cigarette smoking in Madinah, Saudi Arabia: A school-based cross-sectional study. BMC Public Health 2015; 15(1):17

21 Dar-Odeh NS, Aleithan FA, Alnazzawi AA, Al-Shayyab MH, Abu-Hammad SO, Abu-Hammad OA. Factors affecting oral health determinants in female university students: a cross-sectional survey in Saudi Arabia. Int J Adolesc Med Health 2017;32(1):1

22 Al Baik M, Abdrabulnabi A, Aldahan S, Alkhadhrawi N. Electronic cigarette in Saudi Arabia: an online survey. Valley Int J 2014;1:411-426

23 Owusu D, Massey Z, Popova L. An experimental study of messages communicating potential harms of electronic cigarettes. PLoS One 2020;15(10):e0240611

24 Nourwali I, Dar-Odeh N. Pleomorphic adenoma in the lower lip: a case report and a review. Eur J Dent 2019;13(4):649-653

25 Tian Z, Li L, Wang L, Hu Y, Li J. Salivary gland neoplasms in oral and maxillofacial regions: a 23-year retrospective study of 6982 cases in an eastern Chinese population. Int J Oral Maxillofac Surg 2010;39(3):235-242

26 Carlson ER, Odd RA, Textbook and Colour Atlas of Salivary Gland Pathology. Iowa: Wiley-Blackwell; 2008:171-198

27 Kumar A, Popli G, Bhat S, Mohan S, Sowdepalli A, Kumari K. Oral cancer incidence trends in Delhi (1990-2014): an alarming scenario. South Asian J Cancer 2019;8(2):116-119

28 Kumar M, Nanavati R, Modi TG, Dobariya C. Oral cancer: etiology and risk factors: a review. J Cancer Res Ther 2016;12(2):458-463

29 Grafton-Clarke C, Chen KW, Wilcock J. Diagnosis and referral delays in primary care for oral squamous cell cancer: A systematic review. Br J Gen Pract 2019;69(679):e112-e126

30 Gupta B, Johnson NW, Kumar N. Global epidemiology of head and neck cancers: a continuing challenge. Oncology 2016;91(1):13-23

31 Warnakulasuriya S. Global epidemiology of oral and oropharyngeal cancer. Oral Oncol 2009;45(4-5):309-316 